\title{
Fully Regenerative braking and Improved Acceleration for Electrical Vehicles
}

\author{
Wim J.C. Melis, Owais Chishty \\ School of Engineering, University of Greenwich \\ United Kingdom
}

\begin{abstract}
Generally, car brake systems use hydraulic brake technology, which converts the excess of kinetic energy into heat, effectively resulting in an energy loss. Regenerative braking technology focuses on converting this kinetic energy of the decelerating vehicle back into electrical energy that can then be reused for example during acceleration. Current hybrid vehicles are equipped with such regenerative braking technology, which makes them particularly interesting for situations with frequent deceleration, like city traffic. However, the technology used in these vehicles has its limitations and therefore does not stand on its own, but is always assisted with conventional hydraulic brakes. This paper looks at removing this limitation and allowing a vehicle to fully rely on regenerative braking technology to deal with any braking situation ranging from simple slow down to emergency stops. To enable this, multiple generators with different gear ratios are used. The additional benefit of this construction is that, by introducing the appropriate control circuit, the generators can be used as electrical engines. Since these motors are connected with different gear ratios there is a more consistent acceleration at any speed. The paper shows that the overall efficiency of the system is very close to the efficiency of the generators used while achieving braking performance similar to conventional braking mechanisms.
\end{abstract}

\section{Introduction}

The energy crisis has been around for quite a while and the situation is unlikely to improve in the near future. Hence, there have been ever increasing efforts in determining different technologies to improve fuel efficiency. One of these trends has been a return to electrical vehicles, currently mainly through hybrid vehicles [1]. In either of these cases, electrical motors are used for the propulsion of the vehicle. One of the main reasons that these motors are considered beneficial is related to their high overall efficiency [2]. The actual impact on the environment, however, largely depends on the environmental impact of the actual generation of the electricity [3]. Another benefit of electrical vehicles is that the actual motor can also be used as a generator, which is in itself exploited in hybrid vehicles that use this principle to convert kinetic energy back into electrical energy during deceleration and braking. However, most current hybrid vehicles are equipped with a single electrical motor [4], which has certain limitations. Since a generator will only be effective within a certain rpmrange, there is a limited spectrum within which kinetic energy will be converted into electrical energy. Consequently, it is impossible for this generator to deal with all possible braking situations. Considering that, especially during city traffic, the amount of energy lost in deceleration is quite large there is a need to look into recovering this energy for future use. Therefore, this paper investigates how one can build a system that fully relies on regenerative braking technology for any type of deceleration.

Regenerative braking technology has been around for quite a while [5], however most people have looked at a single generator setup [6] and they did certainly not consider the requirement to deal with all possible braking conditions so that regenerative braking would be the only braking mechanism for a vehicle [5]. When all braking situations are considered then the system should be able to deal with a minor slow down situation up to a full emergency braking in order to fully replicate the functioning of the current hydraulic brakes.

The paper starts with giving a description of the problems faced when using regenerative braking as the only braking mechanism, followed by the proposed design to overcome these problems in the next section and an overview of the control mechanism that allows using the generator as motor and vice versa. A test-rig of the proposed design was built and used to test the mechanism, the results of which are presented in the next section, after which the paper concludes.

\section{Proposed Design}

\subsection{Design Principles}

Current regenerative braking systems use only one generator. When this generator is connected to a 
load, then it will extract kinetic energy from the system and convert it into electrical energy. If the braking capacity of the generator is large enough in correspondence with the mass of the vehicle, then switching the generator on/off will lead to shocks in the driving experience.

Currently braking is done with a pedal that allows for a continuous range of braking, going from very minor slow down to full emergency stops. This continuous range is far from the simple on/off that a single generator can offer. In order to obtain this continuous range with a generator-based system, one could reduce the amount of load presented to the generator. Presenting a variable load to the generator could be done by using a variable resistor, but that would mean that all energy gathered is turned into heat, as with the hydraulic brake systems. The purpose of using a generator is to recover the energy and store it in either a battery or a super-capacitor. When batteries are used as a load, they are generally equipped with a battery-charging unit, which not only protects the batteries from over charging but also presents a continuous load to the circuit connected with it. Therefore, in order to present a variable load to the generator, the load is being connected with the generator on a pulse basis. This quick switching behavior automatically averages itself and therefore allows for a variety of different loads being presented to the generator. The load being presented to the generator now depends on the percentage of time that the load is connected within a certain time period. Figure 1 shows an example of three different switching pulses together with the average load that is presented to the generator.

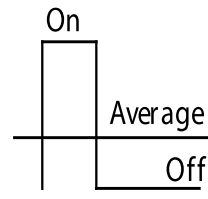

(a)

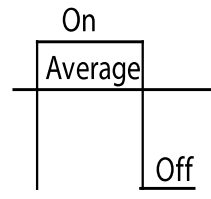

(b)

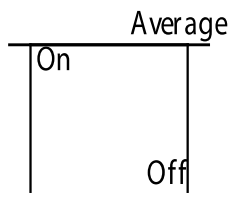

(c)
Figure 1. Pulsing the connection to the load changes the average load to the generator.

An additional problem lies in the fact that if regenerative braking is used as the only braking mechanism, then it also needs to cope with full stop situations and emergency braking. A generator is most efficient for a certain rpm, which means that once it is below this threshold value it will no longer generate electrical energy. Consequently, the generator will no longer extract kinetic energy from the system. In practice this would mean that the vehicle slows down to a certain speed and then would have to "roll-out", which is not acceptable. To overcome this problem, multiple generators were introduced, each connected to the shaft with a different gear ratio. Using these different gear ratios allows for the fact that there is at least one generator operating efficiently, independent of the speed of the vehicle. Consequently, efficient braking is possible up to a full emergency stop. Considering the set of generators and the fact that they are all efficient at different points in time, while needing to be pulsed to ensure they are connected to an appropriate load a control circuit is required to deal with these requirements.

\subsection{Control Circuit}

The requirements for the control circuit is that it needs to take in a continuous input from the brake pedal, and connect the generators to the load, depending on the required amount of braking. The first design [7] was based on using a microcontroller in combination with a set of relays. The relays would be used to connect the load to the generator, whereas the microcontroller would be driving the relays in a pulsed manner based on the braking requirements. The problem with using relays is that they have a limited lifetime as in relation to switching and would wear out particularly quickly due to the continuous pulsing/switching that they undergo. Considering that the purpose of the design is to build a reliable system that allows for fully regenerative braking, another switching mechanism was required. Since the current from generator to load could be large a power electronics solutions with BJTs was designed. The design uses a double H-bridge [8] per motor/generator (See Figure 2). Whereas one $\mathrm{H}$ bridge is used for the motor functionality, the second is used for the generator function. The H-Bridge allows for the motor to turn either direction, which allows the vehicle to move forward as well as backward and brake respectively to the direction it is traveling in. Each vertical branch of the H-bridge has a set of BJTs in parallel with a relay in series. The relay is of the normally closed type and is generally not switched. The reason for this combination of components per branch is to improve the circuit reliability. As much as a relay, also a BJT can fail and this may result in either a permanently open or closed connection. If the connection is permanently open, then the parallel BJT can take over temporarily, if the BJT no longer switches off, then the relays can be used to disconnect that path. In each of the cases, the control circuit could detect such failure by measuring the current that is used to drive the BJT, since the base current determines whether the transistor is conducting or not. A full summary of the relationship between a particular transistor/relay branch being switched on and the respective operation can be found in Table 1 . The microcontroller is programmed in such a way that there is a short time period during which there is no connection when switching from one mode to another as to prevent short-circuiting any of the components. 
Using BJTs for fast switching introduces an additional challenge. A BJT is namely likely to go into saturation when being switched on, which prevents it from being switched off quickly. This behavior would remove the effect of the fast pulsing to reduce the overall load presented to the generator and therefore also change the brake experience. Therefore, the control circuit for each of the BJTs is enhanced with a Baker Clamp [9], which applies negative feedback through the use of a variety of diodes to prevent charge from being stored on the BJT's base. Consequently the BJTs can be switched on and off very quickly as required.

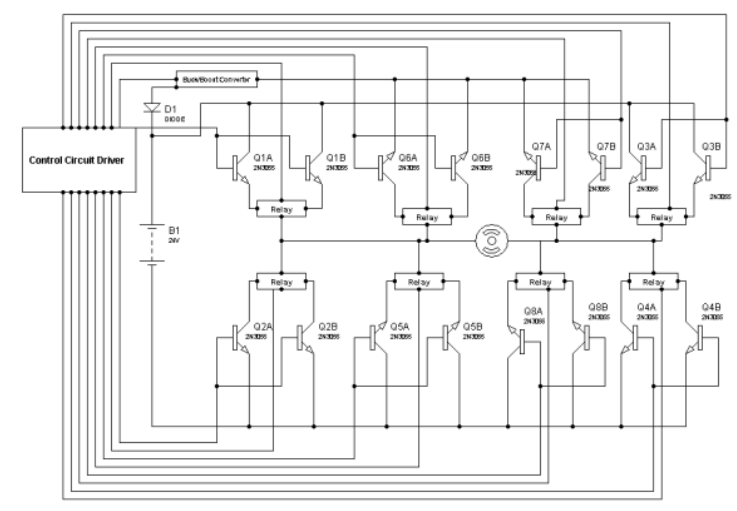

Figure 2. Double H- bridge circuit to control motor and generator functionality

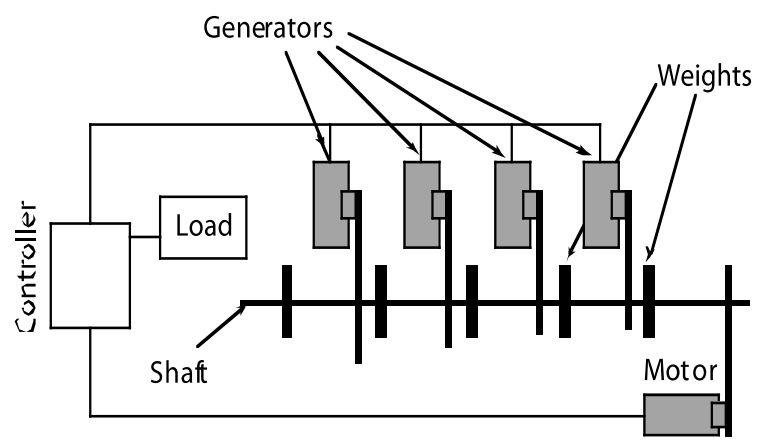

Figure 3: Block Diagram of Prototype Setup

Table 1. H-bridge circuit function description

\begin{tabular}{|c|c|c|c|c|c|c|c|c|}
\hline B1 & B2 & B3 & B4 & B5 & B6 & B7 & B8 & Description \\
\hline On & Off & Off & On & Off & Off & Off & Off & $\begin{array}{c}\text { Motor runs } \\
\text { Clock Wise }\end{array}$ \\
\hline Off & On & On & Off & Off & Off & Off & Off & $\begin{array}{c}\text { Motor runs } \\
\text { Anti-Clock } \\
\text { Wise }\end{array}$ \\
\hline Off & Off & Off & Off & On & On & Off & On & $\begin{array}{c}\text { Braking, } \\
\text { Once Motor } \\
\text { acts as } \\
\text { Generator in } \\
\text { Clock Wise } \\
\text { direction }\end{array}$ \\
\hline & & & & Off & $\begin{array}{c}\text { Braking, } \\
\text { since Motor } \\
\text { acts as } \\
\text { Generator in } \\
\text { Anti-Clock } \\
\text { Wise } \\
\text { direction }\end{array}$ \\
\hline
\end{tabular}

\subsection{Calculations for the Prototype System}

The prototype was designed for use on a trike, which would have a maximum speed of $30 \mathrm{~km} / \mathrm{h}$ and a total maximum weight of $120 \mathrm{~kg}$. In order to determine the amount of energy that could be in the system, one needs to start by looking at the speed and the resulting force related to this. The speed itself is: Max Speed $=30 \frac{\mathrm{km}}{\mathrm{h}}=8.33 \frac{\mathrm{m}}{\mathrm{s}}$, which is then converted into rpm as follows: $30 \frac{\mathrm{km}}{\mathrm{h}} 8.10 \frac{\mathrm{rpm}}{\mathrm{km} / \mathrm{h}}=243 \mathrm{rpm}$. When the trike is at maximum speed, it will go up to $8.33 \mathrm{~m} / \mathrm{s}$ and in an emergency brake situation, it would need to decelerate to $0 \mathrm{~m} / \mathrm{s}$, hence the total force would be: $F=m a=120(8.33-0) / 10=100 \mathrm{~N}$, assuming that the vehicle should stop in $10 \mathrm{~s}$. When the trike has 26 inch wheels $(r=d / 2=0.6604 / 2=$ $0.3302)$, then that would mean that the torque is: $\tau=F r=100 * 0.3302=33.02 \mathrm{Nm}$. This means that the overall mechanical load is: $P_{\text {Mech }}=\tau \omega=$ $33.02\left(243 * \frac{2 \pi}{60}\right)=840 \mathrm{Watt}$. According to British Standards, the electrical power is $25 \%$ higher than the mechanical power, which results in: $P_{\text {Electrical }}=840 * 1.25=1050 \mathrm{~W}$. In order to deal with the need for different gear ratios, this project uses 4 × $250 \mathrm{~W}$ motor/generators for its prototype, leading to the overall system as shown in Figure 3. Each of the motors was of type MY1016 by Unite Motors, and had the characteristics as specified in Table 2 in relation to torque and rpm. The four generators were connected to the central shaft with gear ratios of $0.5,1,1.5$ and 3 for generators 1 to 4 respectively. Due to technical difficulties to build the system onto the trike, the prototype was eventually constructed as a separate test rig that was driven by an electrical motor, with a maximum rpm of 130, and the weight for the system was simulated by adding weights onto the shaft with a total value of $30 \mathrm{~kg}$.

Table 2. Motor Specification

\begin{tabular}{|c|c|}
\hline RPM & Torque \\
\hline 3400 & 0.1 \\
\hline 3300 & 0.2 \\
\hline 3200 & 0.3 \\
\hline 3100 & 0.4 \\
\hline 3000 & 0.5 \\
\hline 2900 & 0.6 \\
\hline 2800 & 0.7 \\
\hline 2700 & 0.8 \\
\hline 2600 & 0.9 \\
\hline
\end{tabular}




\section{Results}

\subsection{Braking}

Due to the difficulties experienced with mounting the system onto the trike and the prototype had to be constructed as a test rig. Therefore, the system was over-designed in comparison to the limitations of the test rig, and this in two aspects. The first aspect is the fact that the design could deal with rpms up to 243 , whereas the motor on the test rig could only provide up to $130 \mathrm{rpm}$. Secondly, the system only had weights up to $30 \mathrm{~kg}$, whereas it was originally designed for a total maximum weight of up to $120 \mathrm{~kg}$. Both of these limitations prevented the system from being tested at full specification. However, in a real vehicle one would rarely be encountering these maximum values. A consequence of these smaller values lies in the fact that the actual timings are very small and therefore the measuring becomes more difficult and is prone to a larger error. Nevertheless, the following results do serve the purpose of indicating the operation of the system. Before any brake test was performed, the system was run for at least $15 \mathrm{~s}$ so that it would get to full speed.

Table 3. Time for Regenerative braking with/without load

\begin{tabular}{|c|c|c|}
\hline \multirow{2}{*}{$\begin{array}{c}\text { Generators } \\
\text { Used }\end{array}$} & \multicolumn{2}{|c|}{ Time to Completely Stop (s) } \\
\cline { 2 - 3 } & No Load & With Load \\
\hline 1 & 1.80 & 3 \\
\hline $1 \& 2$ & 1.65 & 2.7 \\
\hline $1,2 \& 3$ & 1.35 & 2 \\
\hline $1,2,3 \& 4$ & 0.70 & 1.3 \\
\hline
\end{tabular}

A first measurement tests the time for a complete brake with and without load, meaning before and after the actual weights were mounted. The results of this measurement when using different generators for braking are shown in Table 3 . Within this table, one can notice that the values decrease gradually as more generators are used for the braking, which is as expected. One can also notice that using all generators is not 4 times more efficient than using a single generator, which is related to the fact that not all generators run at their optimal efficiency due to the different gear ratios with which they are connected to the shaft. The difference between loaded and unloaded clearly shows that if the system is loaded with weights, then it will take longer to extract the kinetic energy. Putting these numbers in perspective for the vehicle it was designed for, one needs to consider that the actual rotational speed would be approximately double and the weight/load up to four times larger. Taking these two factors into account, one can notice that the brake time would still be reasonable and close to the value used in the calculation of the system, namely $10 \mathrm{~s}$. The overall performance of this regenerative braking is also very close to that of conventional braking mechanisms.

A second test was performed to specifically determine the advantage of pulsing the connection between generator and load. The purpose of this pulsing is to obtain a variable load for the generator and therefore ensure a smoother braking experience. Therefore, this test compares the difference between no pulsing, short and long pulsing and the influence on required braking time for full stop. The results for this test are show in Table 4. The results in this table indicate that the pulsing mechanism ensures a different braking time if the pulsing is longer and therefore the required more gradual braking is achieved.

Table 4. Time For Regenerative Braking With Varied Levels of Pulsing the Load

\begin{tabular}{|c|c|}
\hline Pulsing Level & Time to completely stop (s) \\
\hline None & 1.9 \\
\hline Long ON pulse & 2.2 \\
\hline Short ON pulse & 2.5 \\
\hline
\end{tabular}

The second introduced mechanism, of using different gear ratios was also tested separately to evaluate the influence of the gear system. This test was performed by bring the system to $89 \mathrm{rpm}$ and then switching one generator on completely to brake the system. The results for this test are shown in Table 5. As can be seen in Table 5, generator 4 stops the system in about half the time required by the second generator. This is due to the gear ratio for generator 4 being large, which allows the generator to remain in its optimal rpm for a much longer period of time, consequently, ensuring a more efficient and hence faster braking.

Table 5: Time for Regenerative braking with different gear ratios

Generator Used Time to completely stop(s)

\begin{tabular}{|c|c|}
\hline Generator Used & Time to completely stop(s) \\
\hline 2 & 1.5 \\
\hline 4 & 0.725 \\
\hline
\end{tabular}

\subsection{Acceleration}

One of the major benefits besides the actual energy recovery is the reversibility of the system itself. Considering that the motor/generators are connected to the shaft with different gear ratios, that also ensures a better acceleration at a variety of speeds. This is shown by the results in Table 6 , which indicate that for a larger gear ratio, the system is brought up to speed (50 rpm) much faster. In the same line, this also means that if the system is already at a certain speed then another motor will be able to provide more torque and hence accelerate the vehicle faster. This can also be deducted from looking at the specification of the motor as provided 
in Table 1 and combining it with the gear ratios, as shown in Figure 4. This graph shows that the larger the gear ratio, the larger the torque at low rpm, and vice versa, resulting in a more continuous torque across the full speed range. Hence, by choosing the gear ratios appropriately one can ensure proper acceleration and deceleration for the vehicle.

Table 6. Time for the system to reach $\mathbf{5 0}$ RPM using Individual Generators as Motors

\begin{tabular}{|c|c|}
\hline Generator Used & Time to reach $\mathbf{5 0 ~} \mathbf{~ p m ~ ( s ) ~}$ \\
\hline 1 & 6.9 \\
\hline 2 & 5 \\
\hline 3 & 4.6 \\
\hline 4 & 3.9 \\
\hline
\end{tabular}

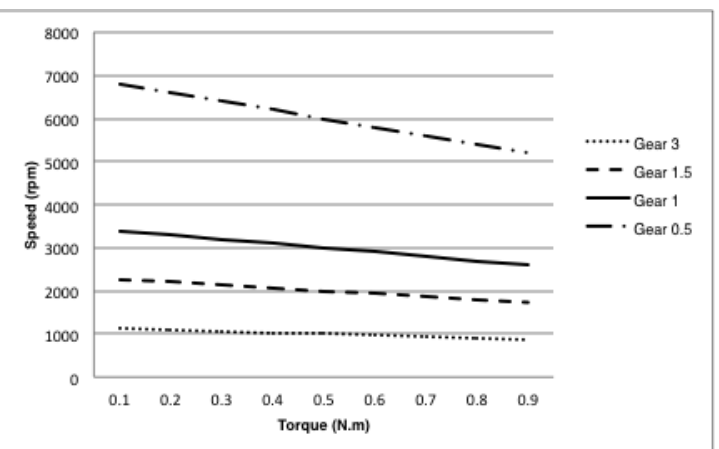

Figure 4: Speed versus Torque for Different Gear Ratios

\subsection{Control Circuit}

The double H-Bridge was also simulated separately in order to test its full functionality together with the program in the microcontroller to ensure a good separation between the different states and therefore prevent any short circuit situations. As can be seen in Figure 5, there are different modes of operation, allowing for the motor to be rotated either direction, and be pulsed if required. The same applies to the generator, which can be used to brake in either direction and pulsed as required to smooth the braking process.

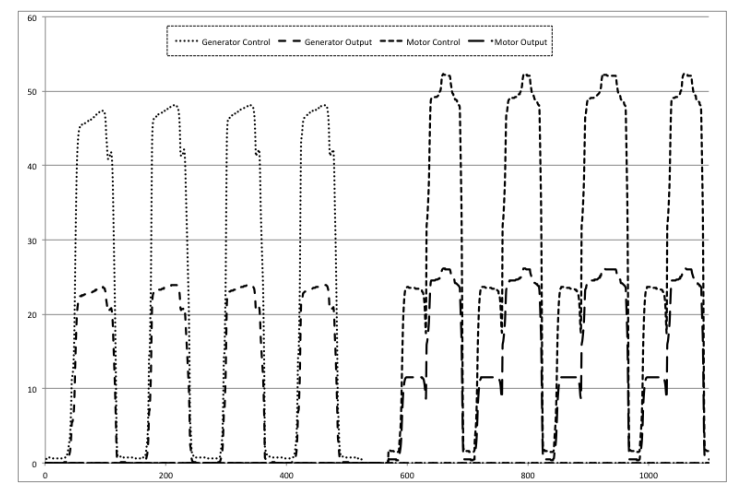

Figure 5: Motor/Generator Control Simulation

\section{Conclusion}

Regenerative braking allows for a vehicle to recover its kinetic energy when braking. Up to now, there have been no systems that fully rely on regenerative, braking, however this paper presents a method to use regenerative braking for all kinds of deceleration. The energy gathered during the deceleration can then be used again during acceleration. The proposed system allows a smooth braking experience while also dealing with full stop and emergency brake situations. The smooth braking experience is achieved by controlling the connection between the generator and its load. Full braking is achieved by using multiple generators, each connected to the shaft with a different gear ratio. This setup ensures that there is always one generator working efficiently and therefore extracting kinetic energy from the system.

Additionally, the system can be reversed when the generators are used as motors to drive the vehicle. Since each motor is connected to the shaft with a different gear the system benefits from an improved acceleration independent of vehicle speed. The switching between both functionalities can be achieved by using a double $\mathrm{H}$-Bridge, which has been expanded with extra circuitry for reliability and to allow for fast switching speeds.

The measured results indicate that the pulsing mechanism ensures a smooth braking experience, as well as the gear system taking care of easing the deceleration from any speed to stand still. Although the measured results were taken on a prototype system that had certain limitations in comparison to the original design, one can easily notice that the braking times for the designed system would be quite in line with existing braking technologies with the additional benefit of energy recovery. During the conversion from kinetic to electrical and vice versa there are minor efficiency losses, which are in line with the efficiency of electrical motors/generators used.

The two principles presented in this paper could also be applied to existing systems. Considering that most existing systems only have a single generator a choice would need to be made to use either of the proposed techniques. So, one could use the pulsing mechanism or the gear system. In this case, the better option probably lies in the gear system, since it provides benefits also for acceleration. However, it tends to come at a higher cost.

\section{References}

[1] Yoong, M.K. (2010) 'Studies of regenerative braking in electric vehicle', in Proceedings of the IEEE Conference on Sustainable Utilization and Development in Engineering and Technology, p. 40-41. 
[2] Binesti, D., (1996) 'Core Losses and Efficiency of Electrical Motors using New Magnetic Materials', in IEEE Transactions on Magnetics, 32 (5), p. 4887-4889.

[3] Ji, S., Cherry, C. R., Bechle, M. J., Wu, Y., Marshall, J. D. (2012) 'Electric Vehicles in China: Emissions and Health Impacts' in Environmental Science and Technology, 46(2), p. 2018-2024.

[4] Ceuca, A., Tulbure, A., Risteiu, M. (2010) 'The Evaluation of Regenerative Braking Energy' in Proceedings of the 16th International Symposium for Design and Technology in Electronic Packaging, p. 65-68.

[5] Broadbent, H.R., Beasley, J., Turrall, J.C. (1962) 'Regenerative braking for multiple-unit stock' Journal of the Institute of Electrical Engineers, 8 (90), p. 298-300.

[6] Suntharalingam, P. (2010) 'Effect on regenerative braking efficiency with deceleration demand and terrain condition', in Proceedings of the 5th IET International Conference on Power Electronics, Machines and Drives (PEMD 2010), p. 1-6.

[7] Chishty, O. and Melis, W. J.C. (2012) 'Energy Regeneration from Decelerating Vehicle' in Proceedings of the World Congress on Sustainable Technologies (WCST), p. 19-22.

[8] Haskew, T.A. and Hill, E.M., (1999). 'Regeneration mechanisms in a DC motor with an H-bridge inverter.' In Proceedings of the International Conference on Electric Machines and Drives (IEMD), p. 531-533.

[9] Quek, D. and Yuvarajan, S. (1994) 'A Novel Gate Drive for the MCT Incorporating Overcurrent Protection.' In Conference Record of the Industry Applications Society Annual Meeting, Vol. 2, p. 1297-1302. 